\title{
Involvement of the arginine repressor in lysine biosynthesis of Thermus thermophilus
}

\begin{abstract}
Correspondence
Makoto Nishiyama

umanis@mail.ecc.u-tokyo.ac.jp
\end{abstract}

Received 15 June 2006

Revised 23 August 2006

Accepted 30 August 2006

\author{
Kei Fujiwara, ${ }^{1}$ Taishi Tsubouchi, ${ }^{1}$ Tomohisa Kuzuyama ${ }^{1}$ \\ and Makoto Nishiyama ${ }^{1,2}$ \\ ${ }^{1}$ Biotechnology Research Center, the University of Tokyo, 1-1-1 Yayoi, Bunkyo-ku, Tokyo \\ 113-8657, Japan
}

${ }^{2}$ RIKEN Spring-8 Center, 1-1-1 Kouto, Sayo-cho, Sayo-gun, Hyogo 679-5148, Japan

\begin{abstract}
Lysine biosynthesis of Thermus thermophilus proceeds in a similar way to arginine biosynthesis, and some lysine biosynthetic enzymes from $T$. thermophilus so far investigated have the potential to function in arginine biosynthesis. These observations suggest that arginine might regulate the expression of genes for lysine biosynthesis. To test this hypothesis, the $\arg R$ gene encoding the regulator of arginine biosynthesis was cloned from $T$. thermophilus and its function in lysine biosynthesis was analysed. The addition of arginine to the culture medium inhibited the growth of an arginase gene knockout mutant of $T$. thermophilus, which presumably accumulates arginine inside the cells. Arginine-dependent growth inhibition was not alleviated by the addition of ornithine, which is a biosynthetic intermediate of arginine and serves as a peptidoglycan component of the cell wall in $T$. thermophilus. However, the growth inhibition was cancelled either by the simultaneous addition of lysine and ornithine or by a knockout of the $\arg R$ gene, suggesting the involvement of $\arg R$ in regulation of lysine biosynthesis in $T$. thermophilus. Electrophoretic mobility shift assay and DNase I footprinting revealed that the ArgR protein specifically binds to the promoter region of the major lysine biosynthetic gene cluster. Furthermore, an $\alpha$-galactosidase reporter assay for this promoter indicated that arginine repressed the promoter in an argR-dependent manner. These results indicate that lysine biosynthesis is regulated by arginine in $T$. thermophilus.
\end{abstract}

\section{INTRODUCTION}

Two pathways are known for lysine biosynthesis: the diaminopimelate pathway found in most bacteria and plants, and the $\alpha$-aminoadipate (AAA) pathway found in fungi and yeasts. Our recent studies revealed that the extremely thermophilic bacterium Thermus thermophilus synthesizes lysine through AAA. The pathway differs from the known AAA pathway found in lower eukaryotes. The Thermus pathway starts from the condensation of 2oxoglutarate with acetyl-CoA to synthesize homocitrate, and proceeds in a manner similar to that in lower eukaryotes until the step of AAA synthesis (Kobashi et al., 1999; Kosuge \& Hoshino, 1999). However, the subsequent part of the pathway is totally different from that in lower eukaryotes, but similar to ornithine synthesis from glutamate, a step in arginine biosynthesis (Fig. 1) (Miyazaki et al., 2001, 2002).

Amino acid biosynthesis is usually controlled by an end product in the pathway by two different systems: inhibition of a biosynthetic enzyme and repression of its gene

Abbreviations: AAA, $\alpha$-aminoadipate; EMSA, electrophoretic mobility shift assay; TCA, tricarboxylic acid. expression. In most cases, a single end product controls its own biosynthesis. However, in the lysine biosynthesis of T. thermophilus, all enzymes so far analysed were found to be able to catalyse the reactions in the tricarboxylic acid (TCA) cycle and arginine biosynthesis (Miyazaki et al., 2001, 2002, 2003, 2004; Wulandari et al., 2002), and 2-oxoglutarate, which is provided through the TCA cycle, is a precursor of both arginine and lysine. This suggests that lysine biosynthesis of $T$. thermophilus is regulated by arginine as well as lysine. Homocitrate synthase (Hcs), which catalyses the first reaction in the pathway, is strictly inhibited by lysine (Wulandari et al., 2002). In addition to the inhibition by lysine, we also found moderate inhibition of Hcs by arginine (Wulandari et al., 2002). As for transcriptional control, we recently demonstrated that the promoter of the homocitrate synthase gene (hcs promoter), which is the promoter of the major lysine biosynthetic gene cluster, is regulated by lysine through leader-peptide-mediated transcriptional attenuation (Tsubouchi et al., 2005). A remaining question is whether the hcs promoter is also regulated by arginine. In this paper, we report the involvement of ArgR, a global arginine regulator, in the transcriptional regulation of the hcs promoter, depending on the availability of arginine. 


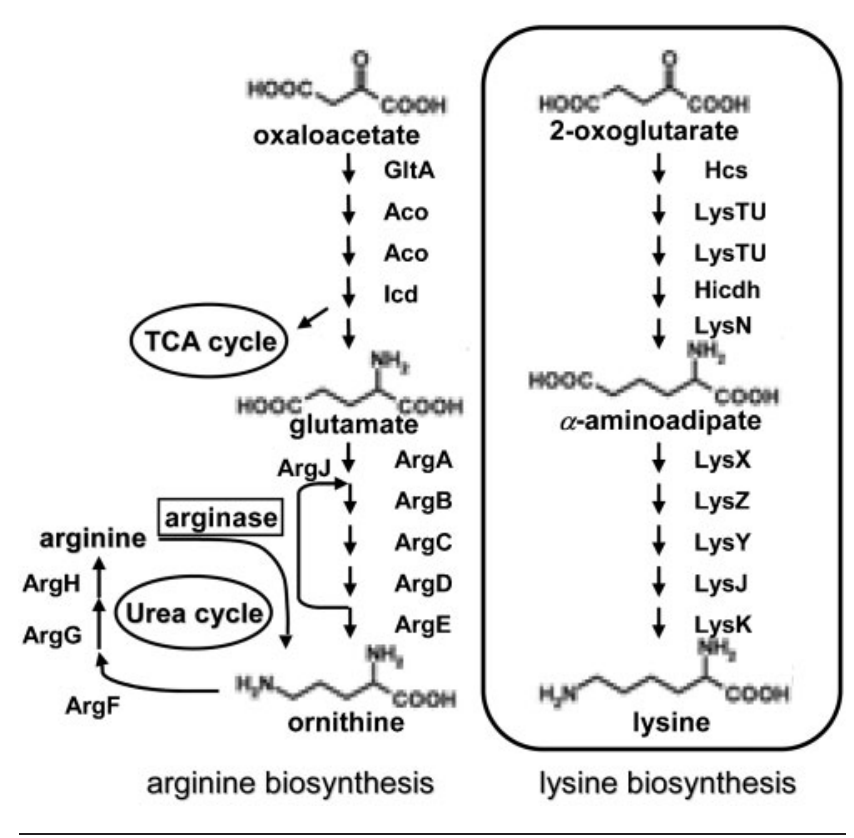

Fig. 1. Lysine biosynthetic pathway of $T$. thermophilus (right) and related metabolic pathways. Enzymes responsible for the corresponding reactions in the TCA cycle and arginine metabolism are shown as gene products in $E$. coli in most cases. Arginase, which catalyses the conversion of arginine to ornithine, is boxed.

\section{METHODS}

Bacterial strains and growth media. T. thermophilus TSU130 (a mutant of the agaT gene encoding $\alpha$-galactosidase derived from $T$. thermophilus HB27) was constructed by methods described previously (Fridjonsson \& Mattes, 2001; Fridjonsson et al., 2002), and used as the host for the reporter assay. T. thermophilus cells were cultivated in TM medium (Koyama et al., 1986) or MM medium (Tsubouchi et al., 2005) supplemented with or without various additions. Escherichia coli JM105 [endA1 thi rpsL sbcB15 hsdR4 $\triangle\left(\right.$ lac-proAB)/F' $\operatorname{traD} 36$ pro $A B$ lac $\left.I^{\mathrm{q}} Z \Delta \mathrm{M} 15\right]$ was used for DNA manipulation, and E. coli BL21(DE3) $\left[\mathrm{F}^{-}\right.$ompT hsdSB $\left(\mathrm{r}_{\mathrm{B}}^{-} \mathrm{m}_{\mathrm{B}}^{-}\right) d c m$ gal $\lambda(\mathrm{DE} 3)]$ was used as the host for gene expression. For the cultivation of E. coli, $2 \times$ YT medium, containing Bactotryptone $1.6 \%$, yeast extract $1 \cdot 0 \%$ and $\mathrm{NaCl} 0.5 \%$, was generally used. All chemicals were purchased from Wako Pure Chemical, Kanto Chemicals or Sigma. Enzymes for DNA manipulation were purchased from Takara Shuzo.

Knockout of the arginase and $\arg R$ genes in $T$. thermophiIus. The nucleotide sequences of oligonucleotides used in this study are shown in Table 1. The plasmids for the knockout of arginase were constructed as follows. Two independent PCRs using ANSM1/ ANSM2 for amplifying $500 \mathrm{bp}$ of a $5^{\prime}$-portion of the arginase gene TTC1132 of T. thermophilus HB27 and ANSU1/ANSU2 for amplifying $500 \mathrm{bp}$ of a $3^{\prime}$-portion of the arginase gene as primers were performed with $T$. thermophilus TSU130 chromosomal DNA as the template. After the amplified $5^{\prime}$ - and $3^{\prime}$-fragments had been cloned into pT7Blue (Novagen), the resulting plasmids were digested with $X b a \mathrm{I} / E c o$ RI and HindIII/HincII, respectively. Two fragments were cloned into pBluescript II SK(+) (Stratagene) to yield pRNSMU. An additional PCR for amplifying the heat-stable hygromycin B

Table 1. Oligonucleotides used in this study

\begin{tabular}{|c|c|}
\hline Name & Sequence \\
\hline ANSM1 & 5'-CGCTCTAGATGGCGCCTCCTTCGGTGCCCATAG-3' \\
\hline ANSM2 & 5'-CCAGAATTCATGGGGGGCATGGTAGCAGAAACG-3' \\
\hline ANSU2 & 5'-CGGGTCGACTCCСCСТAAGCCTCCTCCTCATG-3' \\
\hline ANSCN-F & 5'-GGAGGTGGCCTGTGGTGATCGCAAGCCCTT-3' \\
\hline HygR-R & 5'-CCAAGCTTCTATTCCTTTGCCCTCGGACGA-3' \\
\hline HCSUP & 5'-GAGGAGCTACCAGGCCCGGGAGAGGCGGTT-3' \\
\hline HCSDWN & 5'-GCATGCACCCCTCCCCAAAAGAAAACCG-3' \\
\hline RFUP & 5'-GAATTCGCGGCCGCCTTCATAGACCAGTGG-3' \\
\hline RFDWN & 5'-GAATTCCATATGGCCTTAAGCATACCCCGT-3' \\
\hline RRKOU1 & 5'-CTGAATTCAGTGCTGGAGCTCTTTCTCAAG-3' \\
\hline RRKOU2 & 5'-CAGGATCCGTGAAAAGGACCACGGCGAAGC-3' \\
\hline RRKOCN-F & 5'-CTAGACCCGGTGCGCTTCCTCT-3' \\
\hline RRKOCN-R & 5'-TCTTCCAGGTCTAGAGGAGGCC-3' \\
\hline TtArgRN & 5'-GCTCTAGAAAGGAGGTGCATATGGGCAACAAGGCGGAACGGCACCGC-3' \\
\hline TtArgRHisC & 5'-CCCAAGCTTTCAGTGGTGGTGGTGGTGGTGGTGGTGGGAACCGGAAAGCGCCCGCCGCAG-3' \\
\hline CLWT & 5'-CTCGCGGCGTTTCGTATACCCTTACGGGCAAGATG-3' \\
\hline CLWT-C & 5'-CATCTTGCCCGTAAGGGTATACGAAACGCCGCGAG-3' \\
\hline CLMUT & 5'-CTCGCGGCGTTTCGCCTAGGCTTACGGGCAAGATG-3' \\
\hline CLMUT-C & 5'-CATCTTGCCCGTAAGCCTAGGCGAAACGCCGCGAG-3' \\
\hline
\end{tabular}


phosphotransferase gene was performed using HygR-F/HygR-R as the primers and p8S-T31-hph5 (Nakamura et al., 2005) as the template. An amplified fragment was cloned into pT7Blue to produce pHygRT. pRNSMU was digested with EcoRI/HindIII, and the larger DNA fragment was blunt-ended and ligated with the KpnI-HindIII smaller DNA fragment of pHygRT, which was also blunt-ended. The resulting plasmid, pRNMHygU, was used to knock out the arginase gene of T. thermophilus TSU130 by the method described previously (Hidaka et al., 1994). An arginase gene knockout mutant of T. thermophilus TSU130 verified by PCR with a set of primers ANSCN-F/ ANSCN-R was named T. thermophilus ADK1.

The plasmid for the knockout of $\operatorname{argR}$ was constructed as follows. Two independent PCRs using RRKOM1/RRKOM2 for amplifying $446 \mathrm{bp}$ of a $5^{\prime}$-portion of the argR gene and RRKOU1/RRKOU2 for amplifying $446 \mathrm{bp}$ of a $3^{\prime}$-portion of the $\operatorname{argR}$ gene were performed with pBM4K710, carrying the $\operatorname{argR}$ gene previously cloned from $T$. thermophilus HB27 (unpublished), as the template. The amplified $5^{\prime}$ - and 3 '-fragments were cloned into pBluescript II SK( + ) in the same manner as described above except that the fragments were digested with $C l a \mathrm{I} / E c o \mathrm{RI}$ and BamHI/HindIII, respectively. The resulting plasmid, named pWRAB, was digested with EcoRI/HindIII, blunt-ended, treated with calf intestine alkaline phosphatase, and ligated with the blunt-ended HindIII fragment of pUC19-39-442KmR (Maseda \& Hoshino, 1998) containing the heat-stable kanamycin nucleotidyltransferase gene (Liao et al., 1986). The resulting plasmid, named pWRAB-KmRF, was used to knock out the $\operatorname{argR}$ gene of T. thermophilus TSU130 in the same way. Disruption of the $\operatorname{argR}$ gene in the resulting mutant was confirmed by PCR with primers RRKOCN$\mathrm{F}$ and RRKOCN-R. pWRAB was also used to remove the heat-stable kanamycin nucleotidyltransferase gene from the chromosomal DNA of the $\operatorname{argR}$-knockout mutant. Three kanamycin-sensitive colonies were isolated from about 1000 colonies. Deletion of the heat-stable kanamycin nucleotidyltransferase gene was confirmed by PCR with the same primers. The resulting $\arg R$-knockout mutant was named T. thermophilus WRK07. T. thermophilus ADK2 ( $\triangle a r g R, \Delta$ arginase gene) was generated by the knockout of the arginase gene of $T$. thermophilus WRK07 by using pRNMHygU.

Phenotypic analysis of the arginase gene knockout mutant. The arginase gene knockout mutant, ADK1, was cultured for $10 \mathrm{~h}$ in $5 \mathrm{ml} \mathrm{TM}$ medium containing $1.6 \mathrm{mM} \mathrm{CaCl}_{2}$ and $1.6 \mathrm{mM} \mathrm{MgCl}_{2}$. A small portion $(1 \mathrm{ml})$ was taken from the culture, centrifuged at $15000 \mathrm{~g}$ for $30 \mathrm{~s}$, and the precipitate was washed with MM medium three times. Cell suspension equivalent to $10 \mu \mathrm{l}$ culture was added to $5 \mathrm{ml}$ MP medium (MM medium supplemented with $100 \mu \mathrm{M}$ proline) and incubated at $70{ }^{\circ} \mathrm{C}$. Proline was added to $\mathrm{MM}$ medium to accelerate the growth. Growth was analysed by monitoring the $\mathrm{OD}_{600}$ of the culture at appropriate intervals.

Overexpression and preparation of His-tagged ArgR protein. To express the $\operatorname{argR}$ gene in a form with a $\mathrm{His}_{8}$-tag at the $\mathrm{COOH}$ terminus, PCR was performed with the primer pairs TtArgRN and TtArgRHisC, using pBM4K710 as the template. The PCR product with the correct nucleotide sequence was chosen and cloned as an NdeI-HindIII fragment into pET26b $(+)$ (Novagen) to yield pETArgRHis. E. coli BL21(DE3) cells harbouring pET-ArgRHis were precultured in $5 \mathrm{ml} 2 \times$ YT medium with kanamycin at $50 \mu \mathrm{g} \mathrm{ml}^{-1}$ overnight. The culture was transferred to $11 \mathrm{LB}$ medium containing kanamycin at $50 \mu \mathrm{g} \mathrm{ml}^{-1}$. When the culture had grown to an $\mathrm{OD}_{600}$ of 0.6 , IPTG was added at a final concentration of $0.4 \mathrm{mM}$. After cultivation for $14 \mathrm{~h}$ at $25^{\circ} \mathrm{C}$, cells were harvested, suspended in $30 \mathrm{ml}$ STU buffer $(500 \mathrm{mM} \mathrm{NaCl}, 20 \mathrm{mM}$ Tris/HCl pH 8.0, $500 \mathrm{mM}$ urea), disrupted by sonication, and heated at $70^{\circ} \mathrm{C}$ for $20 \mathrm{~min}$. Debris was removed by centrifugation at $40000 \mathrm{~g}$ for $30 \mathrm{~min}$ and phenylmethylsulfonyl fluoride (final $2 \mathrm{mM}$ ) was added to the supernatant. The enzyme solution was applied onto a
Ni-NTA resin (Novagen), and washed with 10 vols STU buffer followed by washing with 2 vols STU buffer containing $20 \mathrm{mM}$ imidazole. Elution was done with STR buffer $(150 \mathrm{mM} \mathrm{NaCl}, 20 \mathrm{mM}$ Tris/ $\mathrm{HCl} \mathrm{pH} 8 \cdot 0,5 \mathrm{mM}$ arginine) supplemented with $0 \cdot 4 \mathrm{M}$ imidazole. The eluted fractions were dialysed against STR buffer, and used as purified ArgR. The purity of ArgR was verified by SDS-PAGE. Protein concentration was determined by the method of Bradford (1976). Gel filtration was performed to estimate the quaternary structure, with a Superose 12HR 10/30 gel exclusion column (GE Healthcare Bio-Sciences) equilibrated in ST buffer with or without $5 \mathrm{mM}$ arginine.

Electrophoretic mobility shift assays (EMSAs). A 205 bp fragment containing the hcs promoter region and a $290 \mathrm{bp}$ fragment containing the $\operatorname{argF}$ promoter region were amplified from $T$. thermophilus TSU130 chromosomal DNA by PCR with primers HCSUP/ HCSDWN and RFUP/RFDWN, and cloned into pT7Blue to yield pHcsUT and pRFUT, respectively. To construct the hcs promoter mutant, two independent PCRs were performed using RPHCSR/ CLMUT and RPHCSF/CLMUT-C as primers, and cloned into pT7Blue. Portions of the two reaction products were mixed together and subjected to additional PCR using RPHCSF and RPHCSR as primers. An amplified fragment was cloned into pT7Blue to produce pHCSUTmut. The hcs promoter region, the mutated hcs promoter region and the $\operatorname{argF}$ promoter region were amplified by PCRs from pHcsUT, pRFUT and pHcsUTmut with primers HCSUP/HCSDWN, HCSUP/HCSDWN and RFUP/RFDWN, respectively. After purification these fragments were endlabelled with $\left[\gamma^{32} \mathrm{P}\right] \mathrm{ATP}$. The reaction

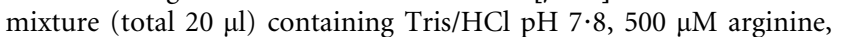
$2 \mathrm{mM} \mathrm{MgCl}_{2}, 1 \mathrm{mM}$ EDTA, $50 \mathrm{mM} \mathrm{NaCl}, 1 \mathrm{mM}$ dithiothreitol, $250 \mu \mathrm{g}$ bovine serum albumin $\mathrm{ml}^{-1}, 10 \%(\mathrm{v} / \mathrm{v})$ glycerol, $1 \%$ sucrose, Nonidet P-40 and $0 \cdot 05 \mu \mathrm{g}$ poly $(\mathrm{dI}-\mathrm{dC}) \mathrm{ml}^{-1}$ was mixed with the labelled probe $(1 \mathrm{nM})$ and $0-400 \mathrm{nM}$ ArgR. Binding of ArgR to the labelled DNA fragment was started by adding the labelled fragment to the reaction mixture preincubated for $10 \mathrm{~min}$ at $70^{\circ} \mathrm{C}$ and the mixture was further incubated at $70^{\circ} \mathrm{C}$ at least $30 \mathrm{~min}$. The reaction mixture was used for $5 \%$ PAGE in TAE ( $40 \mathrm{mM}$ Tris/acetate $\mathrm{pH} 8 \cdot 0,2 \mathrm{mM}$ EDTA) buffer. Arginine was added at $5 \mathrm{mM}$ to the electrophoresis buffer only in the experiment for Fig. 3(a). Band shift on the gel was analysed by an FLA-3000G fluoro/image analyser (Fuji Film). In the competition assay, $2 \cdot 5 \mu \mathrm{M}$ unlabelled probe was added. Each competition probe was prepared by annealing each complement pair of $35 \mathrm{bp}$ nucleotides, CLWT/ CLWT-C and CLMUT/CLMUT-C, by incubation at $95^{\circ} \mathrm{C}$ for $5 \mathrm{~min}, 65^{\circ} \mathrm{C}$ for $5 \mathrm{~min}$ and $37^{\circ} \mathrm{C}$ for $5 \mathrm{~min}$.

Construction of reporter plasmid and $\alpha$-galactosidase assays. After pRFUT was digested with $N d e \mathrm{I} / \mathrm{Not} \mathrm{I}$, the resulting smaller fragment was ligated with pET26-5714/Nde-Not (Tsubouchi et al., 2005) previously digested with NdeI/NotI. An EcoRI-BglII fragment from the resulting plasmid was cloned into the appropriate portion of pOF5714 (Fridjonsson \& Mattes, 2001) to yield pTtRF. T. thermophilus cells harbouring reporter plasmids, pTthcs-plp (Tsubouchi et al., 2005) or pTtRF were grown at $60^{\circ} \mathrm{C}$ in TM medium supplemented with $1.6 \mathrm{mM} \mathrm{CaCl}, 1.6 \mathrm{mM} \mathrm{MgCl}_{2}$ and $50 \mu \mathrm{g}$ kanamycin $\mathrm{ml}^{-1}$ until the mid-exponential phase. Cells were harvested by centrifugation at $15000 \mathrm{~g}$ and washed with MM medium three times. Cells were suspended in MM medium or MP medium containing $50 \mu \mathrm{g}$ kanamycin $\mathrm{ml}^{-1}$ and various additives, and the culture was continued for an additional $24 \mathrm{~h}$. Cells were harvested by centrifugation at $15000 \mathrm{~g}$ for $30 \mathrm{~s}$, washed and suspended in $0 \cdot 1 \mathrm{M}$ HEPES buffer $(\mathrm{pH} 7 \cdot 5)$. Crude extract was prepared by sonication, and debris was removed by centrifugation at $15000 \mathrm{~g}$ for $10 \mathrm{~min}$. Proteins were determined by the method described above. $\alpha$-Galactosidase activity was determined by the methods previously described (Tsubouchi et al., 2005), except that $5 \mathrm{mM}$ 4-nitrophenyl $\alpha$-D-galactopyranoside was used as the 
substrate. One unit of activity was defined as the amount of enzyme required to release $1 \mu \mathrm{mol} p$-nitrophenol $\mathrm{min}^{-1}$, and the specific activity of crude extract was expressed as units (mg protein) ${ }^{-1}$.

DNase I footprinting. DNase I footprinting was performed using non-radioactive probes containing the IRD800 label at the $5^{\prime}$-end. DNA probes were prepared as follows. pHcsUT was used as a template for two PCRs with primers IRD800-labelled T7 promoter primer (MWG-Biotech)/M4p and IRD800-labelled M13-29 primer (Li-Cor)/T7p. These PCRs produced DNA fragments that contain an 188 bp hcs promoter region from -73 to +115 with respect to the transcriptional start point. The DNA fragment also contains an approximately $80 \mathrm{bp}$ or $60 \mathrm{bp}$ extension originating from pT7Blue at the upstream and the downstream ends, respectively, at which the IRD800 label was attached. After purification by Microspin S-400 HR columns (GE Healthcare Bioscience), $50 \mathrm{ng}$ of the resulting DNA was used for DNase I footprinting. Binding of ArgR $(0 \cdot 06-7 \mu \mathrm{M})$ to the DNA fragment was performed as in the EMSA experiments (see above) with minor modification: the total volume was changed to $50 \mu \mathrm{l}$, poly $(\mathrm{dI}-\mathrm{dC})$ was omitted, and the arginine concentration was set to $10 \mathrm{mM}$. DNase I cleavage was done by adding $3 \mu \mathrm{l}$ of a solution containing $160 \mathrm{mM} \mathrm{MgCl}_{2}$ and $0 \cdot 25$ units DNase I. After $1 \mathrm{~min}$ incubation at room temperature, the DNase I reaction was stopped by addition of $50 \mu \mathrm{l} 5 \mathrm{M}$ ammonium acetate and $30 \mathrm{mM}$ EDTA. The DNA was extracted with $100 \mu \mathrm{l}$ phenol and $100 \mu \mathrm{l}$ chloroform, precipitated with ethanol, and washed with $70 \%$ ethanol. The pellet, dissolved in $1 \mu \mathrm{l}$ of formamide loading buffer, was heated at $95^{\circ} \mathrm{C}$ for $10 \mathrm{~min}$, rapidly chilled on ice, and applied to a Li-Cor automated DNA sequencer (model 4000L) using a $6 \%$ KBPlus $41 \mathrm{~cm}$ denaturing sequence gel (Li-Cor) at $50^{\circ} \mathrm{C}$.

\section{RESULTS}

\section{Identification of TTC1194 as the argR gene of T. thermophilus}

The transcriptional regulation of arginine biosynthesis is highly conserved in bacteria: a repressor called ArgR interacts with a specific operator sequence (called the ARG box) in the promoter region (Charlier, 2004; Czaplewski et al., 1992; Dimova et al., 2000; Dion et al., 1997; Larsen et al., 2004, 2005; Lim et al., 1987; Lu et al., 1992; Morin et al., 2003; Tian et al., 1994; Xu et al., 2003). For T. thermophilus, Sanchez et al. (2000) previously suggested that the $\operatorname{argF}$ promoter is regulated by ArgR. For the initial investigation, we aligned the sequences between the $\operatorname{argF}$ promoter region containing the ARG box and the hcs promoter region (Fig. 2a). The result of the alignment suggested the existence of an ARG box in the hcs promoter region, suggesting that the hcs promoter is regulated by ArgR. The whole genome sequence determined for T. thermophilus HB27 (Henne et al., 2004) indicates that T. thermophilus possesses only a single homologue of $\arg R$ as TTC1194. We have already cloned a DNA fragment containing the $\operatorname{argR}$ homologue of T. thermophilus HB27 as a different research project, and therefore used the gene for the following expression experiment.

We constructed a plasmid for the expression of the $\arg R$

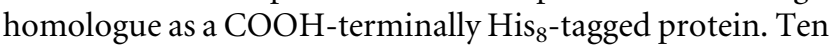
milligrams of the recombinant ArgR homologue was prepared through purification using Ni-NTA resin from (a)
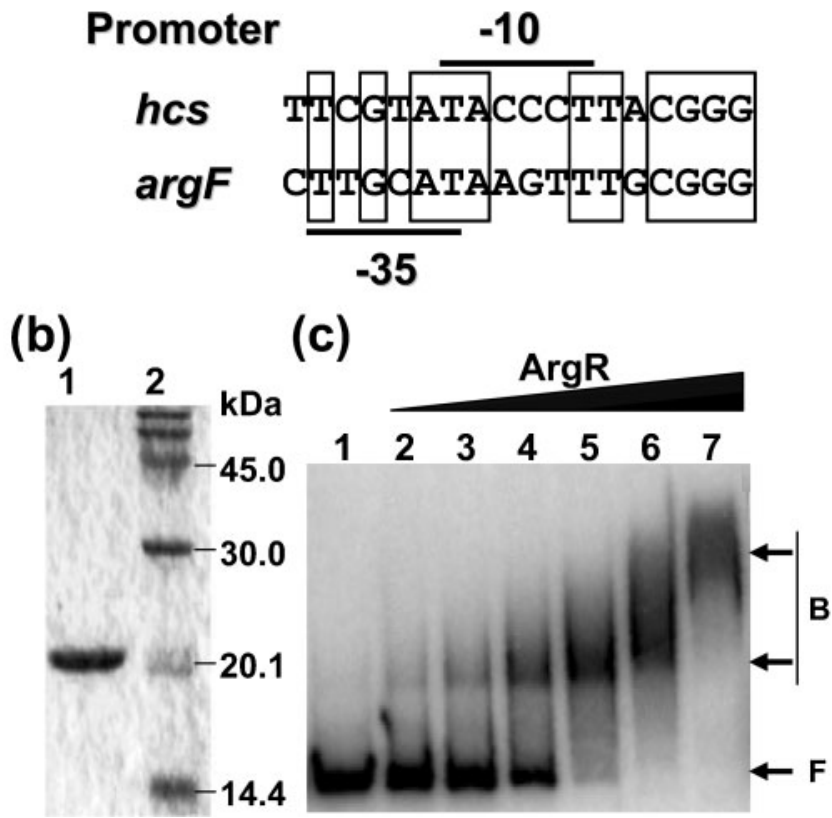

Fig. 2. $\operatorname{ArgR}$ binds to the $\arg F$ promoter DNA. (a) Alignment of the hcs -10 promoter region and a putative ARG box in the $\arg F-35$ promoter region. The hcs promoter -10 region and arg $F$ promoter -35 regions are indicated. Identical nucleotides are boxed. (b) SDS-PAGE of purified ArgR. Lane 1, purified ArgR; lane 2, molecular mass markers: phosphorylase $b$ $(97.0 \mathrm{kDa})$, bovine serum albumin $(66.0 \mathrm{kDa})$, ovalbumin $(45.0 \mathrm{kDa})$, carbonic anhydrase $(30.0 \mathrm{kDa})$, soybean trypsin inhibitor $(20 \cdot 1 \mathrm{kDa})$ and cytochrome $c(14 \cdot 4 \mathrm{kDa})$. (c) EMSA of ArgR for $\arg F$ promoter of $290 \mathrm{bp}$ from $T$. thermophilus. The concentrations of purified ArgR protein added are: lane 1, $0 \mathrm{nM}$; lane 2, $12 \mathrm{nM}$; lane 3, $25 \mathrm{nM}$; lane 4, $50 \mathrm{nM}$; lane 5, $100 \mathrm{nM}$; lane 6, $200 \mathrm{nM}$; lane 7, $400 \mathrm{nM}$. The positions of free DNA (F) and bound DNA (B) are indicated. The assay was performed without arginine in the electrophoresis buffer and the acrylamide gel.

11 of culture with over $95 \%$ purity on SDS-PAGE (Fig. $2 b$ ). We carried out gel filtration to estimate the quaternary structure of the ArgR homologue using a column equilibrated with ST buffer with or without $5 \mathrm{mM}$ arginine. The $\arg R$ homologue encodes an $18 \mathrm{kDa}$ protein with 164 amino acid residues. According to the elution volume, the ArgR homologue has a molecular mass of $60 \mathrm{kDa}$ without arginine, suggesting the configuration of a trimer; however, in the presence of $5 \mathrm{mM}$ arginine the $\operatorname{ArgR}$ homologue was eluted in the fraction with a molecular mass of $100 \mathrm{kDa}$. This result shows that arginine affects the quaternary structure of the $\operatorname{ArgR}$ homologue. ArgR proteins, such as AhrC from Bacillus subtilis (Holtham et al., 1999) and ArgRs from Bacillus stearothermophilus (Dion et al., 1997) and Thermotoga neapolitana (Dimova et al., 2000) are isolated as trimers and are in rapid equilibrium with hexamers. The oligomeric state of some 
(a)

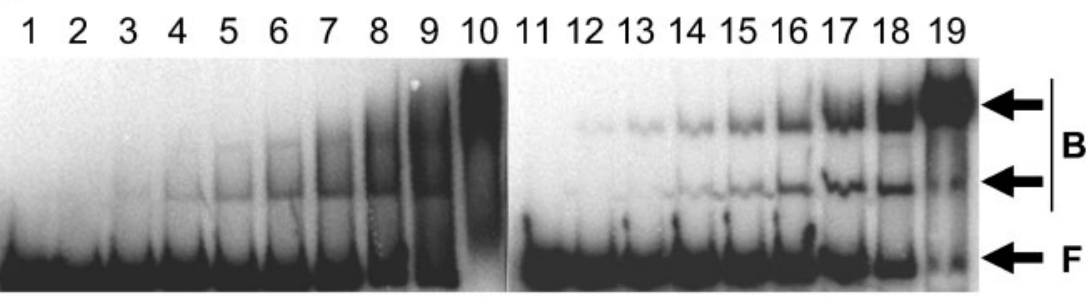

(b)

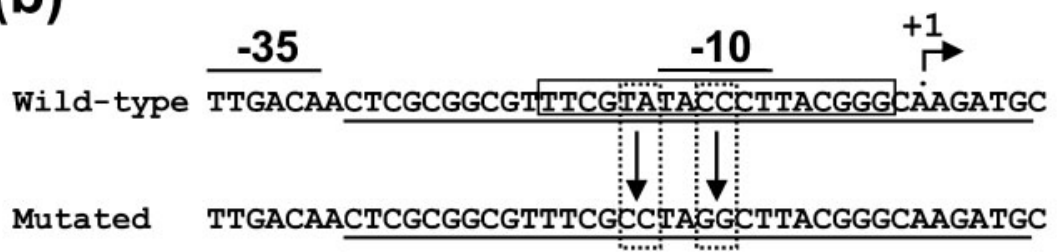

(c)

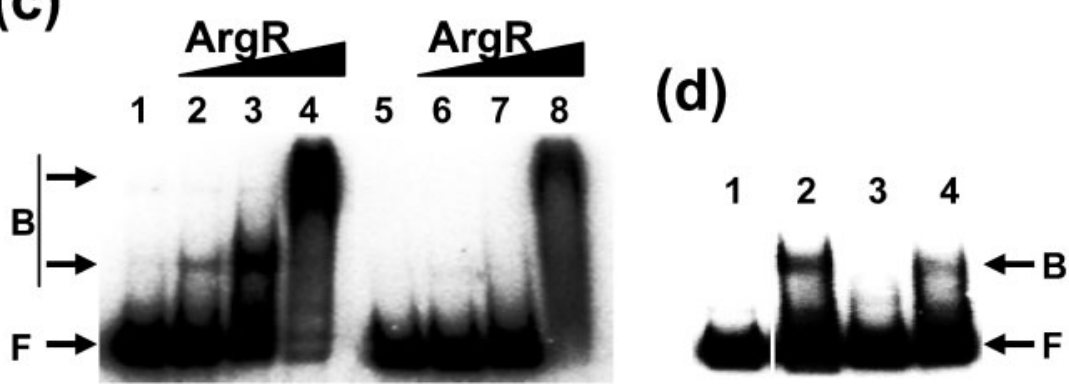

Fig. 3. Specific binding of ArgR protein to the hcs promoter -10 region. (a) EMSA in the presence or absence of arginine in the electrophoresis buffer. Lanes 1-10, $0 \mathrm{mM}$ arginine; lanes 11-19, $5 \mathrm{mM}$ arginine. Concentrations of purified ArgR protein added are: lanes 1 and $11,0 \mathrm{nM}$; lanes 2 and $12,0.8 \mathrm{nM}$; lanes 3 and $13,1.5 \mathrm{nM}$; lanes 4 and $14,3 \mathrm{nM}$; lanes 5 and 15 , $6 \mathrm{nM}$; lanes 6 and 16, $12 \mathrm{nM}$; lanes 7 and 17, $24 \mathrm{nM}$; lanes 8 and 18, $48 \mathrm{nM}$; lanes 9 and 19, $96 \mathrm{nM}$; lane 10, $196 \mathrm{nM}$. (b) Schematic representation of the mutated positions (dotted boxes). Sequences of DNAs added as competition probes are underlined. A putative ARG box is shown by a solid box. (c) EMSA with wild-type DNA region -72 to $+101(+1$ represents the hcs transcriptional start point) (lanes 1-4) or the $4 \mathrm{bp}$-mutated form of this region (lanes 5-8) as the probe. ArgR concentrations are $0 \mathrm{nM}$ for lanes 1 and $5,40 \mathrm{nM}$ for lanes 2 and $6,100 \mathrm{nM}$ for lanes 3 and 7 , and $400 \mathrm{nM}$ for lanes 4 and 8 . The positions of free DNA (F) and bound DNA (B) are indicated. (d) EMSA in the presence of competitor oligonucleotides. Additions are: lane 1, none; lane 2, ArgR; lane 3, 2500-fold excess of unlabelled $35 \mathrm{bp}$ wild-type fragment; lane 4, 2500-fold excess of the unlabelled $35 \mathrm{bp}$ mutated fragment. ArgR concentrations were $0 \mathrm{nM}$ for lane 1 and $100 \mathrm{nM}$ for lanes 2-4. Only the positions of free DNA (F) and fast-migrating complexes of DNA (B) are indicated, because slowmigrating complexes are not observed at $100 \mathrm{nM} \mathrm{ArgR}$ as shown in (c) (lane 3).
ArgR proteins might be concentration dependent and affected by the presence of arginine and/or target DNA. Binding of six arginine molecules per hexamer has been shown for the E. coli and B. stearothermophilus ArgR proteins by X-ray crystallographic analysis (Ni et al., 1999; Van Duyne et al., 1996). Therefore, we assume that the ArgR homologue from $T$. thermophilus is as the hexamer in the arginine-bound form.

To examine binding of the ArgR homologue to the $\arg F$ promoter in vitro, EMSA was performed with DNA fragments covering the $\arg F$ promoter as the probe. The ArgR homologue was able to shift the target probe DNA in a dose-dependent manner with a $K_{\mathrm{d}}$ of approximately 30-50 nM (in monomer equivalent) (Fig. 2c). Then, to confirm that the $\arg R$ homologue is involved in arginine regulation in vivo, we constructed an $\arg R$ homologue knockout mutant (named WRK07) of TSU130, and used it as the host for the reporter assay. In TSU130 harbouring pTtRF, which carries the $\alpha$-galactosidase gene under the control of the $\arg F$ promoter, $\alpha$-galactosidase activity was decreased by the addition of $0.5 \mathrm{mM}$ arginine. In the $\arg R$ knockout mutant WRK07, however, 50-fold increased $\alpha$ galactosidase activity that was not affected by the addition of $0.5 \mathrm{mM}$ arginine was detected (Table 2). Similar deregulated overexpression was observed for arginine regulons in argR mutants of E. coli and Lactococcus lactis (Larsen et al., 2004; Tian et al., 1994). Thus, the $\operatorname{argR}$ homologue is shown to be an $\arg R$ orthologue. We hereafter call the gene $\arg R$. The ArgR protein from $T$. thermophilus shows identity in amino acid sequence to the orthologues of $E$. coli, $B$. stearothermophilus, B. subtilis and Thermotoga neapolitana of $30 \cdot 2,43 \cdot 3,41 \cdot 5$, and $38 \cdot 8 \%$, respectively.

\section{ArgR binds to the hcs promoter region}

ArgR identified above was used for EMSA for the hcs promoter region. EMSA clearly showed that ArgR binds to the hcs promoter region (Fig. 3a). The addition of higher concentrations of ArgR caused the formation of a more slowly migrating complex that might correspond to the hexamer-operator complex, whereas the faster-migrating 
Table 2. Reporter assay for the argF promoter

\begin{tabular}{|c|c|c|c|}
\hline Strain & Genotype & Supplement in MM medium & $\begin{array}{l}\alpha \text {-Galactosidase specific activity } \\
{\left[\mathrm{mU}(\mathrm{mg} \text { protein })^{-1}\right]}\end{array}$ \\
\hline TSU130 & $\Delta a g a T$ & $\begin{array}{l}\text { None } \\
0.5 \mathrm{mM} \text { arginine }\end{array}$ & $\begin{array}{l}2 \cdot 7 \pm 0 \cdot 2 \\
1 \cdot 6 \pm 0 \cdot 2\end{array}$ \\
\hline WRK07 & $\triangle a g a T \Delta \arg R$ & $\begin{array}{l}\text { None } \\
0.5 \mathrm{mM} \text { arginine }\end{array}$ & $\begin{array}{l}132 \cdot 4 \pm 10 \cdot 3 \\
123 \cdot 0 \pm 22 \cdot 1\end{array}$ \\
\hline
\end{tabular}

${ }^{\star}$ Means \pm SD. Three independent experiments were performed.

band might correspond to the trimer-operator complex, as suggested previously by others (Dimova et al., 2000; Morin et al., 2003). When electrophoresis for EMSA was done in electrophoresis buffer containing $5 \mathrm{mM}$ arginine, the slower-migrating band appeared at lower concentrations of ArgR (Fig. 3a). Apparent equilibrium dissociation constants $\left(K_{\mathrm{d}}\right)$ were around $30 \mathrm{nM}$ and $100 \mathrm{nM}$ in the presence and absence of $5 \mathrm{mM}$ arginine in the buffer, respectively. Thus, arginine enhances the affinity of ArgR to the target DNA to some extent. In addition, in the presence of arginine, the complex forms a sharper band compared to the broad and diffuse band observed in its absence, suggesting that the complex is more stable in the presence of arginine. To elucidate the specificity of the binding, we prepared DNA fragments with $4 \mathrm{bp}$ substitutions in the putative ARG box around the hcs promoter -10 region (Fig. 3b), and used them for EMSA. In contrast with the fragments with a wild-type sequence, the $4 \mathrm{bp}$-substituted DNA fragment showed lower affinity to ArgR (Fig. 3c). The observation that no fast-migrating band was formed for the mutant operator suggests that ArgR protein may bind to the mutant ARG box stably only in a hexameric form. In any case, it should be noted that ArgR from T. thermophilus binds to both hcs and $\operatorname{argF}$ promoters with very similar affinity.

To determine the region of ArgR binding more precisely, a 35 bp DNA fragment around the hcs promoter -10 region was used as a competitive probe. The band shifts observed without the competitive probe were diminished by the addition of the $35 \mathrm{bp}$ DNA fragment with the wild-type sequence, whereas the $35 \mathrm{bp}$ DNA fragment with $4 \mathrm{bp}$ mutations had little effect on the shift band of ArgR (Fig. 3d). Furthermore, DNase I footprinting indicated that ArgR protected regions from -23 to -5 for the sense strand and from -19 to -1 for the antisense strand with respect to the transcriptional start point (Fig. $4 \mathrm{a}, \mathrm{b}$ ). These results show that ArgR specifically binds to the $h c s$ promoter -10 region. ArgR also protected the upstream region (site B). This will be discussed below.

\section{Arginine regulates the hcs promoter in vivo}

To elucidate the role of ArgR in the hcs promoter function, we further investigated the effect of arginine on the hcs promoter by reporter gene assay in T. thermophilus TSU130.
T. thermophilus TSU130 cells carrying pTthcs-plp exhibited $\alpha$-galactosidase specific activity of $40 \cdot 9 \pm 7 \cdot 9 \mathrm{mU}(\mathrm{mg}$ protein $)^{-1}$. When a similar assay was performed with the

(a)

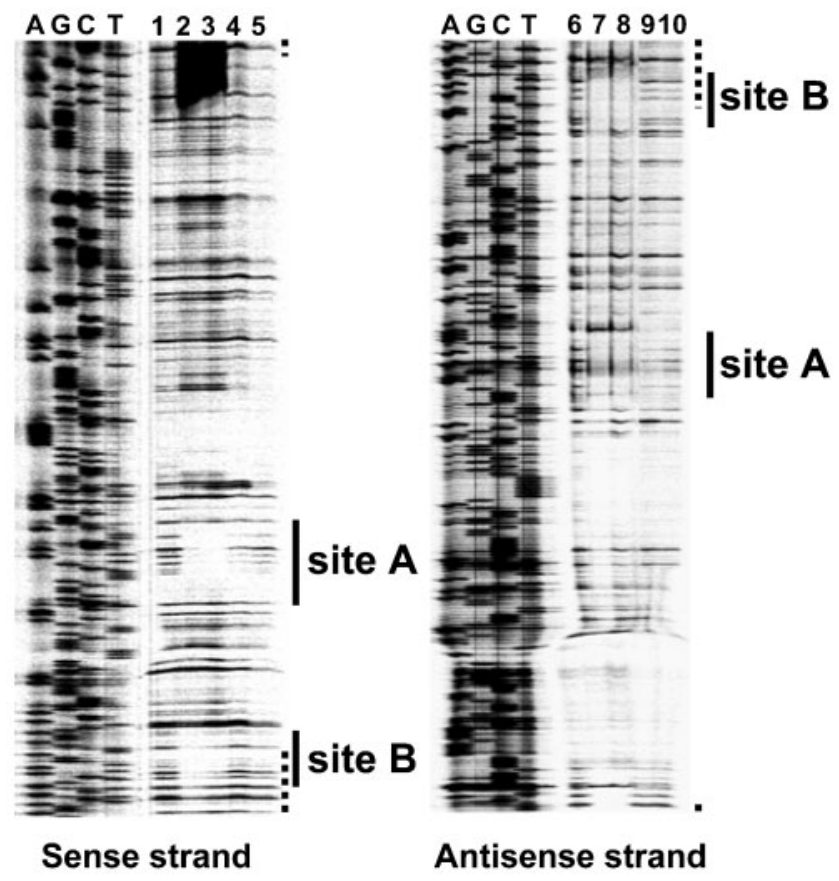

(b)

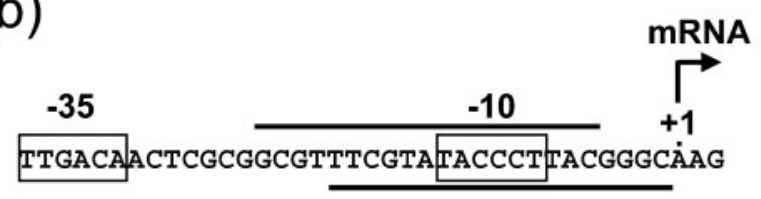

Fig. 4. DNase I footprinting for the hcs promoter region. (a) DNase I footprinting. The concentrations of ArgR protein added are: lanes 1 and $6,0 \mu \mathrm{M}$; lanes 2 and $7,7.0 \mu \mathrm{M}$; lanes 3 and 8, $1.4 \mu \mathrm{M}$; lanes 4 and $9,0.28 \mu \mathrm{M}$; lanes 5 and $10,0.06 \mu \mathrm{M}$. Bars indicate regions protected by ArgR. Dotted lines indicate the sequences derived from pT7Blue. (b) Schematic representation of the regions protected by ArgR (site A). Lower and upper bars indicate regions in the sense strand or antisense strand, respectively, protected by ArgR from DNase I digestion. 
$\operatorname{argR}$ mutant WRK07, the level of $\alpha$-galactosidase activity was increased to $95 \cdot 2 \pm 15 \cdot 2 \mathrm{mU}(\mathrm{mg} \text { protein })^{-1}$, and no enhanced expression [activity 113.4 $\pm 13 \cdot 5 \mathrm{mU}$ (mg protein $)^{-1}$ ] was observed by addition of arginine. From these results, it can be concluded that ArgR is involved in the regulation (repression) of hcs promoter activity.

When $0 \cdot 1 \mathrm{mM}$ arginine was added to the culture of $T$. thermophilus TSU130 carrying pTthcs-plp, the response to arginine varied, with large deviation (data not shown). This observation suggests that a subtle change in culture conditions affects the hcs promoter in $T$. thermophilus TSU130. Considering that arginine degradation in cells might be the possible main reason for the large deviation of the response, we constructed an arginase gene knockout mutant, T. thermophilus ADK1, of T. thermophilus TSU130 and used it as the host for the same reporter assay. As expected, in the arginase-minus genetic background, a reproducible response to arginine was observed. In ADK1, with the knockout of the arginase gene, the $\alpha$-galactosidase activity $\left[36 \cdot 6 \pm 1 \cdot 2 \mathrm{mU}(\mathrm{mg} \text { protein })^{-1}\right]$ obtained in the absence of arginine was decreased threefold [to $\left.11 \cdot 9 \pm 3 \cdot 4 \mathrm{mU}(\mathrm{mg} \text { protein })^{-1}\right]$ by addition of arginine. On the other hand, when a different mutant, ADK2, defective in both $\operatorname{argR}$ and arginase genes was constructed and used as the host for the reporter assay, $\alpha$-galactosidase activity of $113 \cdot 2 \pm 16 \cdot 5 \mathrm{mU}$ (mg protein) ${ }^{-1}$ was obtained in the absence of arginine. Addition of arginine did not decrease $\alpha$-galactosidase activity but increased the activity twofold, to $234 \cdot 2 \pm 10 \cdot 4 \mathrm{mU}(\mathrm{mg} \text { protein })^{-1}$.

\section{Growth inhibition of the arginase knockout mutant by arginine}

The above results indicate the involvement of ArgR in hcs promoter activity. These results also suggest that $T$. thermophilus may show a lysine-auxotrophic phenotype when arginine is added to the culture, due to repression of hcs promoter activity. To examine this possibility, growth of T. thermophilus TSU130, ADK1 and ADK2 was monitored.

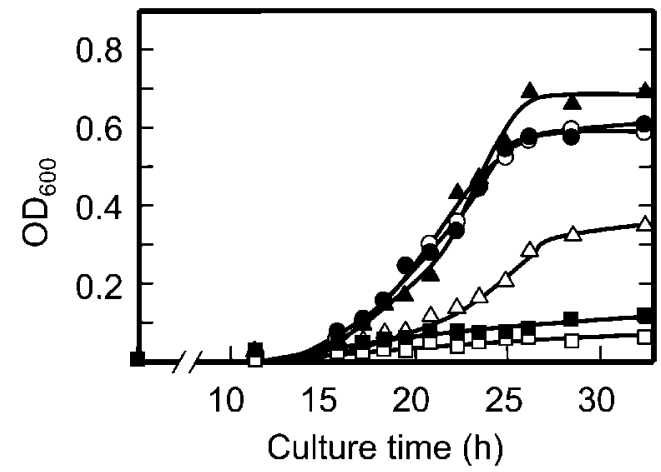

Fig. 5. Growth inhibition of the arginase-knockout mutant by arginine. Growth curves are shown for the wild-type TSU130 $(\bigcirc)$ and the arginase-deficient mutant ADK1 (O) in MP medium. Growth of ADK1 in MP medium supplemented with $0.1 \mathrm{mM}$ arginine $(\square)$, both $0.1 \mathrm{mM}$ lysine and $0.1 \mathrm{mM}$ arginine $(\triangle)$, both $0.1 \mathrm{mM}$ ornithine and $0.1 \mathrm{mM}$ arginine ( $\boldsymbol{\square})$, and all three amino acids (lysine, arginine and ornithine, $0 \cdot 1 \mathrm{mM}$ each) $(\mathbf{A})$ is also shown.

As expected, the growth of the arginase mutant $T$. thermophilus ADK1 was inhibited by the addition of arginine to the minimal medium (Fig. 5), which contrasted with the cases of the wild-type strain $T$. thermophilus TSU130 and the mutant ADK2, defective in both arginase and $\arg R$ genes, where no inhibition by arginine was observed (Table 3). Ornithine is an intermediate of arginine biosynthesis, and its synthesis is therefore controlled by arginine. Furthermore, ornithine is a peptidoglycan component in T. thermophilus (Quintela et al., 1995). We anticipated that an increase in the intracellular arginine pool could activate the ArgR function to repress the arginine biosynthetic gene expression and, as a result, lead to the short supply of ornithine as a cell wall component. We therefore added $0.1 \mathrm{mM}$ ornithine to MP medium, and examined its effect on the growth of the arginase mutant ADK1; however, it had only a slight effect on growth

Table 3. Generation time of ADK1 in MP medium supplemented with arginine, ornithine, and/ or lysine

\begin{tabular}{|c|c|c|c|}
\hline Strain & Genotype & Addition $(0 \cdot 1 \mathrm{mM})$ & Generation time (min) \\
\hline \multirow[t]{2}{*}{ TSU130 } & $\Delta a g a T$ & None & 139 \\
\hline & & Arginine & 106 \\
\hline \multirow[t]{5}{*}{ ADK1 } & $\Delta a g a T$ Darginase & None & 160 \\
\hline & & Arginine & $>300^{*}$ \\
\hline & & Arginine, ornithine & $>300^{*}$ \\
\hline & & Arginine, lysine & 191 \\
\hline & & Arginine, lysine, ornithine & 146 \\
\hline \multirow[t]{2}{*}{ ADK2 } & $\Delta a g a T$ arginase $\Delta$ argR & None & 160 \\
\hline & & Arginine & 158 \\
\hline
\end{tabular}

${ }^{\star}$ Generation times of ADK1 in the presence of arginine, or arginine plus ornithine, could not be determined due to the extremely slow growth. 
(Fig. 5). We next examined the growth of ADK1 in MP medium supplemented with $0 \cdot 1 \mathrm{mM}$ lysine or both $0 \cdot 1 \mathrm{mM}$ lysine and $0 \cdot 1 \mathrm{mM}$ ornithine. The slow growth of ADK1 was partially restored by the addition of $0 \cdot 1 \mathrm{mM}$ lysine, and fully restored by the addition of both $0 \cdot 1 \mathrm{mM}$ lysine and $0 \cdot 1 \mathrm{mM}$ ornithine. When generation times were compared, addition of arginine prolonged the generation time of ADK1 significantly (Table 3 ). The prolonged generation time was partially shortened by addition of lysine and completely normalized by simultaneous addition of lysine and ornithine. On the other hand, the generation time of ADK2, with the knockout of both $\operatorname{argR}$ and arginase genes, was not affected by arginine. These results also support the idea that ArgR regulates lysine biosynthesis in vivo.

\section{DISCUSSION}

In this study, we characterized $\operatorname{Arg} \mathrm{R}$ as a transcriptional regulator for lysine biosynthesis in T. thermophilus. ArgR from $T$. thermophilus was purified as a trimeric protein, and shown to become a hexamer in the presence of arginine. As shown in Fig. 3(a), the bands in the EMSA were somewhat broad and smeary in the absence of arginine, and addition of arginine in the electrophoresis buffer gave clear band profiles. Thus, arginine may assure the formation of a stable ArgR-DNA complex. However, apparent $K_{\mathrm{d}}$ was not so significantly affected by arginine $(30 \mathrm{nM}$ and $100 \mathrm{nM}$ in the presence or in the absence of arginine, respectively). Recently Charlier and colleagues classified arginine regulators into three major types (Charlier, 2004; Morin et al., 2003). Among them, ArgR from Thermotoga, which is the only ArgR classified into class III, is defined as serving as a general regulator exhibiting broad target specificity and low arginine-dependent activation of DNA-binding capacity. In T. thermophilus ArgR, a Gln residue, which is highly conserved in class I ArgR and involved in the highly arginine-dependent activation of DNA-binding ability, is replaced by Ser (Fig. 6). Substitution of Gln for the Ser of ArgR from $T$. neapolitana resulted in a mutant protein exhibiting a marked need for arginine as co-factor and an enhanced target specificity (Morin et al., 2003). It is also shown that the T. thermophilus ArgR is different from other class ArgR proteins in that it can interact with a single ARG box sequence. Arg from T. thermophilus also has a serine residue at the corresponding position and shows low arginine-dependent activation of DNA-binding capacity. The observation that the band shift in EMSA was diminished by addition of a short competitor of $35 \mathrm{bp}$ having only a single ArgR-binding site around the hcs promoter -10 region may also suggest that ArgR interacts with a single ARG box sequence. DNase I footprinting indicates that ArgR binds to the hcs promoter -10 region (site A) as expected. The analysis revealed that another region (site B) was also protected by $\operatorname{ArgR}$ (Fig. 4a, b). However, the protection was found at a region connecting the hcs promoter upstream sequence with the cloning site of the vector pT7Blue. Actually, only a small portion, from -73 to -68 in the sense strand and from -73 to -70 in the antisense strand, was protected for the hcs promoter upstream region, and the other protection was found in the vector region. This observation indicates that the putative ArgR-binding site in the hcs promoter upstream region is artificially generated in our DNase I footprinting experiment. Although the DNase I footprinting could not rule out the possibility that ArgR may bind to other regions which were not tested in this study, our results are consistent with there being a single ARG box covering the hcs promoter -10 region, which regulates hcs promoter activity. From these observations, we may conclude that $\operatorname{ArgR}$ of $T$. thermophilus is a class III regulator.

The addition of arginine inhibited the growth of the arginase gene knockout mutant ADK1 (Fig. 5). Addition of lysine partially overcomes the growth inhibition by arginine, and the simultaneous addition of both lysine and ornithine restores the growth of ADK1 completely, even in the presence of arginine (Fig. 5). It could be that addition of arginine to ADK1 causes diversion of the excess arginine to a pathway that produces a toxic compound and that this pathway is repressed by lysine. However, in both the wildtype and the double mutant, ADK2, defective in arginase and $\arg R$, arginine inhibition was not observed (Table 3). The most likely explanation of these phenomena is that the growth inhibition by arginine in the arginase mutant is attributable to the function of ArgR, therefore suggesting that lysine biosynthesis as well as ornithine synthesis is regulated by ArgR in T. thermophilus. Lysine biosynthesis through AAA is also found in fungi, and AAA synthesis from 2 -oxoglutarate proceeds in the same way in fungi and $T$. thermophilus; however, subsequent synthesis, from AAA to lysine, is totally different between these organisms. The $T$. thermophilus pathway is similar to a step in arginine biosynthesis, while the pathway in fungi starts from adenylation of the $\alpha$-amino group of AAA and contains saccharopine as an intermediate. It should be noted that similar growth inhibition by arginine has been reported in an arginase mutant of the fungus Neurospora crassa, but inhibition is restored by only ornithine (Davis et al., 1970).

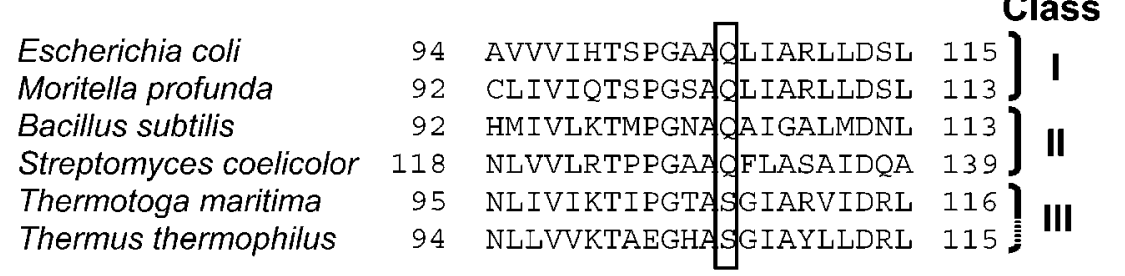

Fig. 6. Partial alignment of ArgR amino acid sequences in three classes. Glutamine residues conserved in class I and II ArgR proteins and serine residues at the corresponding position in class III ArgR proteins are boxed. 
This suggests the presence of a different regulatory system in Neurospora, although it should be taken into account that the fungus has compartmentalization and a specific transport system, and therefore the situation may be totally different.

By EMSA and DNase I footprinting, we demonstrated that ArgR specifically bound to the DNA fragment containing the hcs promoter -10 region (Fig. 3). For further elucidation of the role of ArgR in hcs promoter regulation in vivo, reporter gene assays were carried out. In ADK1, with the knockout of the arginase gene, the addition of arginine repressed the hcs promoter. In ADK2, with the additional mutation of $\arg R$, no repression was observed, whereas the addition of arginine activated the hcs promoter. In WRK07, with an $\arg R$ mutation, no response to arginine was observed. These results may indicate not only the involvement of ArgR in the regulation of lysine biosynthesis in $T$. thermophilus, but also the presence of some other regulatory systems that sense arginine or its metabolites to activate lysine biosynthesis. As described in Results, the involvement of ArgR in the regulation of the hcs promoter was not verified in the parent strain of T. thermophilus; in some cultures, arginine seemed to enhance $\alpha$-galactosidase expression, while it decreased the expression in others. Since such a variable effect was not observed with the addition of other amino acids in the reporter assay, we assume that there are multiple systems to sense arginine and/or its metabolites, and therefore arginine might play a central role in the metabolic flux of related amino acids in $T$. thermophilus. We have very recently found a transcriptional factor, which also regulates lysine biosynthesis, and are analysing its regulatory mechanism (unpublished results). Through detailed research of at least two transcriptional factors, regulatory mechanisms for lysine biosynthesis should be elucidated in the near future.

The lysine biosynthesis of T. thermophilus has an evolutionary relationship with the biosynthesis of arginine and leucine, and the TCA cycle (Nishida et al., 1999). Furthermore, the fact that a similar lysine biosynthesis pathway is also present in archaea (Brinkman et al., 2002; Lombo et al., 2004; Nishida et al., 1999), which are phylogenetically positioned close to the root of the universal tree of evolution (Woese et al., 1990), suggests that lysine biosynthesis was present in the earliest organisms. We may assume that the ancestral enzymes involved in the processes related to current Thermus lysine biosynthesis functioned in lysine and arginine biosynthesis as well as the TCA cycle. In previous studies, we have shown that $T$. thermophilus lysine biosynthetic enzymes have the ability to recognize intermediates of the TCA cycle and arginine biosynthesis as substrates, suggesting that the enzymes have additional roles in those metabolisms even now. It is of interest that an enzyme involved in lysine biosynthesis may play a role in biosynthesis of another basic amino acid, arginine; however, there are other reported cases of such functional overlap. Ledwidge \& Blanchard (1999) showed that the $\operatorname{argD}$ gene encoding $\mathrm{N}$-acetylornithine aminotransferase in the arginine biosynthesis pathway is the most probable candidate for $\operatorname{dapC}$ gene encoding $N$-succinyl-L,L-diaminopimelate:2-oxoglutarate aminotransferase in a major root (DAP pathway) of the lysine biosynthesis pathway in $E$. coli. The $\arg D$ gene is regulated by ArgR (Charlier et al., 1992). However, it is unclear whether this gene is regulated by lysine. In this study, we showed that ArgR, a repressor of arginine biosynthesis, has the ability to regulate lysine biosynthesis in T. thermophilus, although the regulation is apparent in an arginase gene knockout background. Cumulative data on the expression profile of lysine biosynthetic and related genes of $T$. thermophilus will elucidate the complicated regulatory mechanisms managed by multiple transcriptional machinery.

\section{ACKNOWLEDGEMENTS}

We thank Drs A. Nakamura and T. Hoshino (Tsukuba University) for kindly giving us the plasmid p8S-T31-hph5 carrying the heat-stable hygromycin B phosphotransferase gene. This work was supported in part by a grant-in-aid for scientific research from the Ministry of Education, Culture, Sports, Science and Technology of Japan, from Nagase Science and Technology Foundation, and from the Asahi Glass Foundation.

\section{REFERENCES}

Bradford, M. M. (1976). A rapid and sensitive method for the quantitation of microgram quantities of protein utilizing the principle of protein-dye binding. Anal Biochem 72, 248-254.

Brinkman, A. B., Bell, S. D., Lebbink, R. J., de Vos, W. M. \& van der Oost, J. (2002). The Sulfolobus solfataricus Lrp-like protein LysM regulates lysine biosynthesis in response to lysine availability. J Biol Chem 277, 29537-29549.

Charlier, D. (2004). Arginine regulation in Thermotoga neapolitana and Thermotoga maritima. Biochem Soc Trans 32, 310-313.

Charlier, D., Roovers, M., Van Vliet, F., Boyen, A., Cunin, R., Nakamura, Y., Glansdorff, N. \& Pierard, A. (1992). Arginine regulon of Escherichia coli K-12. A study of repressor-operator interactions and of in vitro binding affinities versus in vivo repression. J Mol Biol 226, 367-386.

Czaplewski, L. G., North, A. K., Smith, M. C., Baumberg, S. \& Stockley, P. G. (1992). Purification and initial characterization of AhrC: the regulator of arginine metabolism genes in Bacillus subtilis. Mol Microbiol 6, 267-275.

Davis, R. H., Lawless, M. B. \& Port, L. A. (1970). Arginaseless Neurospora: genetics, physiology, and polyamine synthesis. J Bacteriol 102, 299-305.

Dimova, D., Weigel, P., Takahashi, M., Marc, F., Van Duyne, G. D. \& Sakanyan, V. (2000). Thermostability, oligomerization and DNAbinding properties of the regulatory protein ArgR from the hyperthermophilic bacterium Thermotoga neapolitana. Mol Gen Genet 263, 119-130.

Dion, M., Charlier, D., Wang, H., Gigot, D., Savchenko, A., Hallet, J. N., Glansdorff, N. \& Sakanyan, V. (1997). The highly thermostable arginine repressor of Bacillus stearothermophilus: gene cloning and repressor-operator interactions. Mol Microbiol 25, 385-398. 
Fridjonsson, O. \& Mattes, R. (2001). Production of recombinant $\alpha$ galactosidases in Thermus thermophilus. Appl Environ Microbiol 67, 4192-4198.

Fridjonsson, O., Watzlawick, H. \& Mattes, R. (2002). Thermoadaptation of $\alpha$-galactosidase AgaB1 in Thermus thermophilus. J Bacteriol 184, 3385-3391.

Henne, A., Bruggemann, H., Raasch, C. \& 17 other authors (2004). The genome sequence of the extreme thermophile Thermus thermophilus. Nat Biotechnol 22, 547-553.

Hidaka, Y., Hasegawa, M., Nakahara, T. \& Hoshino, T. (1994). The entire population of Thermus thermophilus cells is always competent at any growth phase. Biosci Biotechnol Biochem 58, 1338-1339.

Holtham, C. A., Jumel, K., Miller, C. M., Harding, S. E., Baumberg, S. \& Stockley, P. G. (1999). Probing activation of the prokaryotic arginine transcriptional regulator using chimeric proteins. J Mol Biol 289, 707-727.

Kobashi, N., Nishiyama, M. \& Tanokura, M. (1999). Aspartate kinaseindependent lysine synthesis in an extremely thermophilic bacterium, Thermus thermophilus: lysine is synthesized via $\alpha$-aminoadipic acid not via diaminopimelic acid. J Bacteriol 181, 1713-1718.

Kosuge, T. \& Hoshino, T. (1999). The $\alpha$-aminoadipate pathway for lysine biosynthesis is widely distributed among Thermus strains. J Biosci Bioeng 88, 672-675.

Koyama, Y., Hoshino, T., Tomizuka, N. \& Furukawa, K. (1986). Genetic transformation of the extreme thermophile Thermus thermophilus and of other Thermus spp. J Bacteriol 166, 338-340.

Larsen, R., Buist, G., Kuipers, O. P. \& Kok, J. (2004). ArgR and AhrC are both required for regulation of arginine metabolism in Lactococcus lactis. J Bacteriol 186, 1147-1157.

Larsen, R., Kok, J. \& Kuipers, O. P. (2005). Interaction between ArgR and AhrC controls regulation of arginine metabolism in Lactococcus lactis. J Biol Chem 280, 19319-19330.

Ledwidge, R. \& Blanchard, J. S. (1999). The dual biosynthetic capability of $\mathrm{N}$-acetylornithine aminotransferase in arginine and lysine biosynthesis. Biochemistry 38, 3019-3024.

Liao, H., McKenzie, T. \& Hageman, R. (1986). Isolation of a thermostable enzyme variant by cloning and selection in a thermophile. Proc Natl Acad Sci U S A 83, 576-580.

Lim, D. B., Oppenheim, J. D., Eckhardt, T. \& Maas, W. K. (1987). Nucleotide sequence of the $\operatorname{argR}$ gene of Escherichia coli K-12 and isolation of its product, the arginine repressor. Proc Natl Acad Sci U S A 84, 6697-6701.

Lombo, T., Takaya, N., Miyazaki, J., Gotoh, K., Nishiyama, M., Kosuge, T., Nakamura, A. \& Hoshino, T. (2004). Functional analysis of the small subunit of the putative homoaconitase from Pyrococcus horikoshii in the Thermus lysine biosynthetic pathway. FEMS Microbiol Lett 233, 315-324.

Lu, C. D., Houghton, J. E. \& Abdelal, A. T. (1992). Characterization of the arginine repressor from Salmonella typhimurium and its interactions with the carAB operator. J Mol Biol 225, 11-24.

Maseda, H. \& Hoshino, T. (1998). Development of expression vectors for Thermus thermophilus. J Ferment Bioeng 86, 121-124.

Miyazaki, J., Kobashi, N., Nishiyama, M. \& Yamane, H. (2001). Functional and evolutionary relationship between arginine biosynthesis and prokaryotic lysine biosynthesis through $\alpha$-aminoadipate. J Bacteriol 183, 5067-5073.
Miyazaki, J., Kobashi, N., Fujii, T., Nishiyama, M. \& Yamane, H. (2002). Characterization of a $l y s K$ gene as an $\operatorname{argE}$ homolog in Thermus thermophilus HB27. FEBS Lett 512, 269-274.

Miyazaki, J., Kobashi, N., Nishiyama, M. \& Yamane, H. (2003). Characterization of homoisocitrate dehydrogenase involved in lysine biosynthesis of an extremely thermophilic bacterium, Thermus thermophilus HB27, and evolutionary implication of $\beta$-decarboxylating dehydrogenase. J Biol Chem 278, 1864-1871.

Miyazaki, T., Miyazaki, J., Yamane, H. \& Nishiyama, M. (2004). $\alpha$ Aminoadipate aminotransferase from an extremely thermophilic bacterium, Thermus thermophilus. Microbiology 150, 2327-2334.

Morin, A., Huysveld, N., Braun, F., Dimova, D., Sakanyan, V. \& Charlier, D. (2003). Hyperthermophilic Thermotoga arginine repressor binding to full-length cognate and heterologous arginine operators and to half-site targets. J Mol Biol 332, 537-553.

Nakamura, A., Takakura, Y., Kobayashi, H. \& Hoshino, T. (2005). In vivo directed evolution for thermostabilization of Escherichia coli hygromycin B phosphotransferase and the use of the gene as a selection marker in the host-vector system of Thermus thermophilus. J Biosci Bioeng 100, 158-163.

Ni, J., Sakanyan, V., Charlier, D., Glansdorff, N. \& Van Duyne, G. D. (1999). Structure of the arginine repressor from Bacillus stearothermophilus. Nat Struct Biol 6, 427-432.

Nishida, H., Nishiyama, M., Kobashi, N., Kosuge, T., Hoshino, T. \& Yamane, H. (1999). A prokaryotic gene cluster involved in synthesis of lysine through the amino adipate pathway: a key to the evolution of amino acid biosynthesis. Genome Res 9, 1175-1183.

Quintela, J. C., Pittenauer, E., Allmaier, G., Aran, V. \& de Pedro, M. A. (1995). Structure of peptidoglycan from Thermus thermophilus HB8. J Bacteriol 177, 4947-4962.

Sanchez, R., Roovers, M. \& Glansdorff, N. (2000). Organization and expression of a Thermus thermophilus arginine cluster: presence of unidentified open reading frames and absence of a Shine-Dalgarno sequence. J Bacteriol 182, 5911-5915.

Tian, G., Lim, D., Oppenheim, J. D. \& Maas, W. K. (1994). Explanation for different types of regulation of arginine biosynthesis in Escherichia coli B and Escherichia coli $\mathrm{K} 12$ caused by a difference between their arginine repressors. J Mol Biol 235, 221-230.

Tsubouchi, T., Mineki, R., Taka, H., Kaga, N., Murayama, K., Nishiyama, C., Yamane, H., Kuzuyama, T. \& Nishiyama, M. (2005). Leader peptide-mediated transcriptional attenuation of lysine biosynthetic gene cluster in Thermus thermophilus. J Biol Chem 280, 18511-18516.

Van Duyne, G. D., Ghosh, G., Maas, W. K. \& Sigler, P. B. (1996). Structure of the oligomerization and L-arginine binding domain of the arginine repressor of Escherichia coli. J Mol Biol 256, 377-391.

Woese, C. R., Kandler, O. \& Wheelis, M. L. (1990). Towards a natural system of organisms: proposal for the domains Archaea, Bacteria, and Eucarya. Proc Natl Acad Sci U S A 87, 4576-4579.

Wulandari, A. P., Miyazaki, J., Kobashi, N., Nishiyama, M., Hoshino, T. \& Yamane, H. (2002). Characterization of bacterial homocitrate synthase involved in lysine biosynthesis. FEBS Lett 522, 35-40.

Xu, Y., Sun, Y., Huysveld, N., Gigot, D., Glansdorff, N. \& Charlier, D. (2003). Regulation of arginine biosynthesis in the psychropiezophilic bacterium Moritella profunda: in vivo repressibility and in vitro repressor-operator contact probing. J Mol Biol 326, 353-369. 